\title{
Tensions and trade-offs in instructional goals for physics courses aimed at en- gineers
}

\section{Andrew Elby, University of Maryland, College Park}

My work focuses on student and teacher epistemologies and how they couple to other cognitive machinery and help to drive behavior in learning environments. My academic training was in Physics and Philosophy before I turned to science (particularly physics) education research. More recently, I have started exploring engineering students' entangled identities and epistemologies.

\section{Eric Kuo, Stanford University}

Dr. Ayush Gupta, University of Maryland, College Park

Ayush Gupta is Research Assistant Professor in Physics and Keystone Instructor in the A. J. Clark School of Engineering at the University of Maryland. Broadly speaking he is interested in modeling learning and reasoning processes. In particular, he is attracted to fine-grained analysis of video data both from a microgenetic learning analysis methodology (drawing on knowledge in pieces) as well as interaction analysis methodology. He has been working on how learners' emotions are coupled with their conceptual and epistemological reasoning. He is also interested in developing models of the dynamics of categorizations (ontological) underlying students' reasoning in physics. Lately, he has been interested in engineering design thinking, how engineering students come to understand and practice design.

Dr. Michael M. Hull, Wayne State College

Assistant Professor of Physical Sciences 


\title{
Tensions and trade-offs in instructional goals for physics courses aimed at engineers
}

\begin{abstract}
In planning and teaching courses for engineering majors, physics instructors grapple with multiple instructional goals: extensive content coverage, quantitative problem solving, conceptual understanding, motivation, and more. The temptation is to treat these goals as mutually reinforcing or at least as not in conflict. We argue, however, that at least for novice instructors, these goals can be in tension. In our study, one instructor was experienced and emphasized traditional quantitative problem solving. A second instructor teaching another lecture section of the same course was a novice who chose to emphasize a goal suggested by physics education research and studies of practicing engineers, namely mathematical sensemaking - translating and seeking coherence between mathematical formalism and physical reasoning. A common final exam, containing standard traditional problems and also opportunities for mathematical sense-making, enabled us to document the following trade-off: the novice instructor outperformed the experienced traditional instructor at fostering mathematical sense-making but underperformed at fostering traditional problem solving. In other words, the novice instructor's success at teaching mathematical sense-making came at a cost. A third instructor, expert in emphasizing mathematical sense-making, showed that it is possible to succeed at teaching mathematical sense-making without a significant trade-off in teaching traditional problem-solving. However, for instructors considering the adoption of physics/engineering education research-based instructional strategies, trade-offs must be acknowledged and tough choices must be made.
\end{abstract}

\section{Introduction}

What instructional objectives in physics courses most help engineering students succeed in their subsequent engineering courses and careers? Faculty often mention "problem solving skills" and "conceptual understanding"; but decades of physics and engineering education research have barely addressed this question empirically. ${ }^{1-3}$

Some engineering educators argue that traditional close-ended, well-structured and well-defined problem-solving of the type demanded by end-of-chapter problems in physics textbooks is important to emphasize, because it develops skills that students can build on and apply in later engineering classes. Others argue that mathematical sense-making - translating and seeking coherence between mathematical formalism and physical reasoning (often intuitive), using mathematics flexibly as part of sense-making about the physical world - is closer to what practicing engineers $\mathrm{do}^{4,5}$. Other faculty tout the importance of additional skills a physics class might teach, such as basic math skills or use of software such as MATLAB.

Historically, most physics courses have emphasized traditional problem solving. The identification of these other possible instructional goals raises the issue of whether and how they should be treated in physics curricula. Given the unchanged time constraints of introductory physics courses, can an instructional target such as mathematical sense-making be emphasized 
without taking away from developing students' traditional problem-solving skills? In this paper, we argue that there can be a trade-off between emphasizing mathematical sense-making and emphasizing traditional problem-solving, both of which we define more carefully below.

Our data come from the first-semester physics course taken by almost all engineering majors at Big University. One section, taught by an experienced, well-regarded professor, emphasized "traditional" quantitative problem solving (Instructor A). Two other sections, one taught by a novice instructor (Instructor B) and the other taught by an instructor experienced with "activelearning" pedagogy (Instructor C), emphasized mathematical sense-making. Our evidence shows that traditional problem solving and mathematical sense-making do not automatically reinforce each other. On a shared final exam, students of the novice instructor who emphasized mathematical sense-making exhibited higher performance on items demanding non-routine mathematical sense-making; but the students expertly instructed in standard quantitative problem solving performed better on traditional quantitative problems. We use these results to argue that, at least initially, physics courses can't "have it all"; tough choices must be made between different instructional goals. Of course, our case study of one course cannot establish this result definitively; our point is to introduce and illustrate the argument to motivate future large- $\mathrm{N}$ studies.

Our results are important because most physics education research (PER) on introductory college/university physics courses does not address such trade-offs and therefore invites the conclusion that a course can have it all. For instance, many studies emphasize how researchbased curricula can lead to greater conceptual gains as measured by instruments such as the Force Concept Inventory. ${ }^{6}$ Few of these studies measure or report on whether the conceptual gains come at the expense of some other goal, such as proficiency at traditional problem solving. The few studies that do address this issue typically report that, as compared to traditionally taught students, students in the reformed courses do just as well on traditional exam problems. ${ }^{7,8}$ And a few studies report that reform-oriented instruction targeted at conceptual understanding also improved students' traditional problem-solving. ${ }^{8}$ But instructor effects might account for some of these results, since more dedicated instructors are perhaps more likely to attempt reforms. Mazur's study at Harvard ${ }^{8}$ avoided this issue, since he gave the same exam to his own students 6 years apart; but the small gains in problem-solving performance he documented (from $63 \%$ to $69 \%$ correct) could have arisen from his improved skill, developed over six years, at teaching problem-solving efficiently, enabling him to spend some class time on Peer Instruction without a trade-off. Furthermore, the lack of any published PER results (that we know of) highlighting a trade-off, such as higher conceptual gains at the expense of lower performance on traditional problems, could result from publication bias. The one near-exception to our generalization that PER studies have not highlighted instructional trade-offs is Hoellwarth et al. ${ }^{9}$ Over three quarters of instruction, the reformed class outperformed the traditional class on conceptual questions. The traditional class and reformed class performed equally well on traditional exam problems in two quarters; bit in the third quarter, the traditional class outperformed the reform class. The authors report, however, that "although we cannot with certainty pin-point the reason why one quarter in three revealed a gap in problem-solving ability, we note that [the third quarter] was the least controlled of the three comparison quarters." Our point is that we know of no published PER studies highlighting an unambiguous trade-off between two instruction goals. 
Speaking more personally as physics and engineering education researchers, we know that some physics faculty will pounce on any trade-off - any "sign of weakness" - as a reason to write off PER-based instruction as watered down and ineffective. It has taken the authors of this paper a long time to view such trade-offs not only as something to discuss forthrightly but as something to study. Sure, an experienced PER expert can achieve multiple instructional goals with minimal trade-offs, as illustrated by one of the instructors in our study and probably by some of the studies just mentioned. But that result is almost irrelevant to a professor considering trying PERbased instructional strategies for the first time.

In the rest of this paper, we will first briefly review literature on (i) expert problem solving of traditional physics problems, (ii) mathematical sense-making in a physics context, and on how those two constructs differ. Then, we present our research questions and methods, highlighting how they operationalize the distinction between traditional problem solving and mathematical sense-making. Next, we present our results, which illustrate the trade-offs mentioned above. Finally, we discuss the implications of these results for engineers and physicists planning and teaching introductory physics courses for engineering majors.

\section{Literature review: Exemplary traditional problem solving vs. mathematical sense-making}

In this section, we briefly present a PER consensus vision of expert quantitative problem solving of close-ended physics problems. We then illustrate what we mean by mathematical sensemaking and how it can differ from that consensus vision.

Expert "traditional" problem-solving. Building on research about differences between novices and experts in solving physics problems, ${ }^{10-12}$ textbooks and researchers - most notably the Physics Education Research and Development group at the University of Minnesota-have articulated prescriptions for students to follow when approaching a physics problem. ${ }^{13,14}$ We call this vision of expert problem solving "traditional" both because it is a refinement of strategies that experienced physics teachers have been teaching for decades and because it is optimized for the types of problems traditionally found in physics textbooks. The prescriptions aim to steer students away from common novice approaches such as identifying the unknown and searching for an equation that contains it, or pattern matching based on superficial aspects of the physical scenario. $^{12,15}$ Although details differ, the prescriptions generally consist of steps like these:

1. Using diagrams as needed, visualize and make sense of the physical situation conceptually/qualitatively.

2. Explicitly identity the relevant physics principles.

3. Using the insights from (1) and (2), write equation(s) that can be solved for the relevant variable(s).

4. Do the needed mathematical manipulations to solve those equations.

5. Do a reality/plausibility check on the final answer.

In short, there's a consensus that expert traditional problem solving involves thinking conceptually and carefully before diving into equations, and then using conceptual knowledge, direct experience, etc. to check the results after completing the mathematical manipulations. 
Previous studies indicate that novices skip or shortchange steps 1-2 and step 5. ${ }^{12,15,16}$ However, instruction that models and rewards this more expert approach can measurably increase students' use of steps $1-2$ and 5 . $^{14}$

Such an approach to problem-solving could help students in their engineering sciences classes, where close-ended quantitative problems are common. This is why improving students' traditional problem-solving skills is a reasonable instructional target for introductory physics courses aimed at engineering majors.

Mathematical sense-making. In expert traditional problem solving, as just noted, conceptual reasoning occurs before the mathematical manipulations, to identify the needed equations, and after the manipulations, to check the result. Conceptual/qualitative reasoning need not occur during step 4 above. By contrast, in mathematical sense-making, as we define it, conceptual reasoning also occurs during the manipulation and inspection of equations or other mathematical objects, revealing a solution pathway that does not include explicit calculation. An example from a previous study of ours illustrates what we mean. ${ }^{17}$ In clinical interviews, after asking students questions about the kinematics equation $v=v_{0}+a t$, we had students consider the following problem:

Suppose you are standing with two tennis balls... You throw one ball down with an initial speed of 2 meters per second; at the same moment, you just let go of the other ball, i.e., just let it fall. I would like you to think aloud while figuring out what is the difference in the speed of the two balls after 5 seconds - is it less than, more than, or equal to 2 meters per second? (Students are told to neglect wind resistance if they bring it up)

The typical, good solutions we analyzed fit into the mold of the expert, "traditional" problem solving approach outlined above: Many students drew diagrams to carefully distinguish what's happening to the dropped vs. thrown ball, identified the constancy of acceleration of freely falling objects near the Earth's surface as the relevant principle (though not always explicitly), wrote $v=v_{0}+a t$ for the two balls separately, solved for each ball's speed at $t=5 \mathrm{~s}$ (obtaining 50 $\mathrm{m} / \mathrm{s}$ and $52 \mathrm{~m} / \mathrm{s}$ ) and then subtracted those two final speeds to find that the thrown ball is moving $2 \mathrm{~m} / \mathrm{s}$ faster than the dropped ball.

Some students, by contrast, also relied on $v=v_{0}+$ at but reasoned in terms of the conceptual meaning of that equation, as follows: Both balls speed up at the same rate due to gravity, and therefore both balls gain the same speed during the fall. Therefore, since the thrown ball starts out $2 \mathrm{~m} / \mathrm{s}$ faster than the dropped ball, the thrown ball is still moving $2 \mathrm{~m} / \mathrm{s}$ faster at the later time.

This second solution relies on mathematical sense-making in two ways. First, in the context of a physics problem that can be solved with a calculation, these students chose to avoid an explicit calculation by adaptively drawing on a heuristic, conceptual method to keep track of the difference in speeds. Second, this conceptual explanation was tied to the mathematical equation, in this case by reading off and exploiting the conceptual meaning of the equation as something like "how fast you end up is how fast you start plus how much you speed up while accelerating." 


\section{Physics instruction emphasizing mathematical sense-making}

We now briefly describe some elements of the learning environments created by Instructors B and $\mathrm{C}$, who emphasized mathematical sense-making. Instead of encouraging students to address problems by following a fixed set of steps, they encouraged students to approach problems in a flexible, adaptive manner, combining common-sense reasoning, conceptual reasoning, quantitative reasoning, and symbolic manipulations - and trying to reconcile among them.

Here is a homework problem, written by the experienced PER-savvy instructor but also used by the novice instructor, designed to scaffold students in seeking this kind of reconciliation:

Standing on a cliff, I take one rock and throw it straight up at a speed of $30 \mathrm{~m} / \mathrm{s}$. I take another rock and throw it straight down at $30 \mathrm{~m} / \mathrm{s}$. Suppose the cliff is 50 meters high.

a) Just based on common sense, which rock would be moving faster when it hits the ground, 50 meters below? What's the reasoning for that?

b) Now find an answer based on the kinematics of constant acceleration: Find $x(t)$ and $v(t)$ for each of the rocks, and find their respective speeds when they hit the ground 50 $m$ below the point of release.

c) Did your answer to (b) agree with your answer to (a)? If not, try to reconcile the contradiction: Figure out what it is about the reasoning in part (a) or part (b) that doesn't work. Get it all to make sense!

In part (a), students commonly answer that the rock thrown down hits the ground with more speed, perhaps thinking that the downward throw is more "direct" or that the downward force of the throw adds to the velocity. In part (b), students use a formal calculation to see that, in fact, both rocks hit the ground with the same speed. Part (c) asks students to do more than recognize the failure of their part (a) intuition. It also prompts students to interrogate both their intuition and the calculation in order to build coherence between the two. The homework solutions point out the key intuition that needs refinement: a ball initially travelling $30 \mathrm{~m} / \mathrm{s}$ upward will return to its initial height travelling $30 \mathrm{~m} / \mathrm{s}$ downward. So the rock thrown up goes up, but when it comes down, it's travelling at the same speed as the rock that is thrown down, and therefore both rocks land with the same speed.

In brief, part (c) explicitly invites students to reconcile common sense and their mathematical reasoning, privileging neither above the other and encouraging students to interpret and make sense of that math instead of just manipulating the equations "plug and chug" style. Students who understand the conceptual point of part (c) will be able to leverage this understanding in the future to answer similar kinematic questions without explicit computation. With this problem, and problems like it throughout the course, the instructors support mathematical sense-making over execution of traditional problem-solving procedures.

Midterm exams rewarded this type of reconciliation. For example, consider this essay question from the first midterm in the novice instructor's class: 
A picture of the monkey-hunter setup from the class demonstration is shown. The monkey is released at the same time as the bullet is launched. Suppose the bullet is launched with an initial speed so that it hits the monkey before the monkey reaches the ground. A rough sketch of the bullet's trajectory is shown on the picture. As you can see, the bullet reaches the peak of its motion before hitting the monkey.

(a) At the instant the bullet reaches the peak of its trajectory, is the monkey at the same

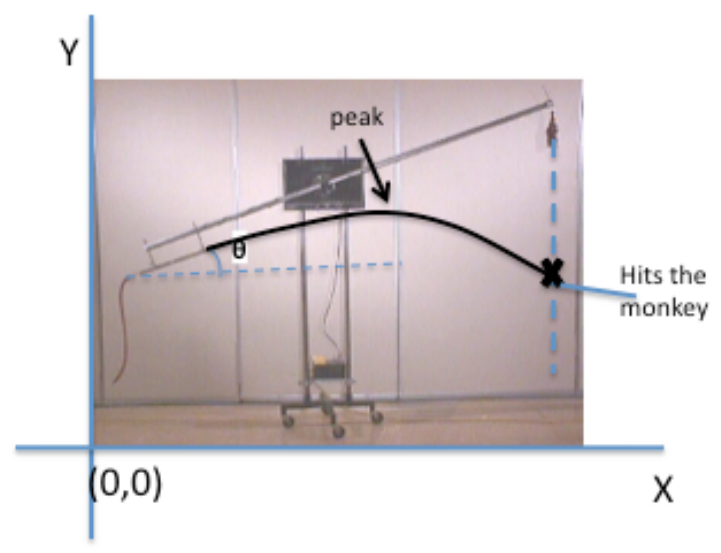
height as the bullet, above it, or below it? Explain your reasoning.

(b) Give a different answer someone else might believe and the reasoning that person would use to support it.

(c) Explain what is wrong with the reasoning in part (b)

Answering (a) and (b), a student might argue that the monkey starts above the bullet and the vertical distance between them keeps narrowing until the point of impact, in which case the monkey is still above the bullet at the peak of the bullet's motion. Or a student might incorrectly overgeneralize from the classic "shot vs. dropped" physics experiment where a ball shot horizontally and a ball dropped from the same height remain at the same height as they approach the ground. A student thinking in this way could conclude that, since the bullet is travelling horizontally at its peak (while the monkey is analogous to the dropped ball in the classic experiment), and since the monkey and bullet are the same height when they hit each other, they must share the same height when the bullet reaches its peak.

To decide which argument is correct (in this case the first argument) and explain why the other is wrong, the student needs to work out some of the mathematics or make an argument similar in form to the "shortcut" approach to the problem about the dropped vs. thrown ball from the Literature Review above: At the peak of the ball's motion, its vertical velocity is zero whereas the monkey has a downward velocity. Since both objects gain downward velocity at the same rate, but the monkey starts with more downward velocity, the monkey always has more downward velocity than the bullet. Therefore, the monkey must always be above the bullet until impact; if the monkey and bullet ever shared the same height at some instant prior to impact, the monkey's greater downward speed would ensure that the bullet passes above the monkey instead of striking it. To complete the resolution of why this physical scenario is not analogous to the classic "shot vs. dropped" experiment, a student could recognize that at the moment the bullet is moving horizontally, the monkey is not dropped from rest, but is instead already moving downwards.

In these and other problems throughout the course, these instructors aimed to help students make sense of the equations and formal mathematical calculations. To help students develop this type 
of reasoning, these instructors used clickers, regularly having students discuss problems of this type in small groups during lecture. In discussion (recitation) sections, students also worked in small groups on such problems.

Why did the instructors emphasize mathematical sense-making so strongly? One reason is that we were conducting a quasi-experiment to gauge the effects of this kind of instruction on students' beliefs about what counts as learning and understanding physics. ${ }^{17}$ Furthermore, as we argued previously, from the perspective of "thinking like a physicist," the mathematical sensemaking shortcut to the tossed vs. thrown ball problem is more insightful/expert than a solution obtained from good traditional problem-solving. ${ }^{17}$ A few researchers have argued that the kind of flexible, adaptive use of mathematics and facile mixing of conceptual and more symbolic reasoning instantiated by mathematical sense-making is essential for engineers as well. ${ }^{4,5}$

Given the lack of consensus about whether traditional problem-solving or mathematical sensemaking is the more important instructional target, our first instinct is to deny the existence of a trade-off: "let's emphasize both!" And indeed, an experienced, PER-savvy instructor can succeed at helping students employ good traditional problem solving and mathematical sensemaking, as our data show. For a more novice instructor, by contrast, the trade-off can be real. That is our main point, and that is what our data will illustrate.

\section{Methods and Research Questions}

In order for our research questions to make sense, we first need to describe classroom context and participant selection.

Participant selection. Big University includes a large, well-regarded college of engineering. Almost all of these students take "Physics 1" our first-semester calculus-based physics course, which focuses on the mechanics of motion. Since Physics I is a core requirement for engineering majors, most students were $1^{\text {st }}$ or $2^{\text {nd }}$ year undergraduates. Our data collection took place during one semester. Three lecture sections were offered, taught by three different instructors. Students' decisions about which section to take generally depend on scheduling constraints, and students were not informed that the different sections would be taught in different ways. (We note, however, that generally positive course reviews were available on-line for the two experienced instructors. No reviews were available for the novice instructor.) Each lecture section enrolled $\sim 140$ students.

Instructor A was an experienced, "traditional" professor, by which we mean he did not employ "active learning" instructional techniques such as clickers, tutorials in discussion sections, etc. His homework consisted of end-of-chapter problems from the textbook and his exam problems were similar to those. Instructor B was a relatively novice physics education researcher who had previously taught one physics course to $\sim 20$ pre-service elementary school teachers, using "active learning" pedagogy, but who had never taught a large lecture-based introductory physics course. Instructor $\mathrm{C}$ was a long-time physics education researcher and experienced instructor. Both $\mathrm{A}$ and $\mathrm{C}$ were well-regarded instructors with a history of positive course reviews. All three instructors were male. 
At the beginning and end of the semester, students were invited to complete a survey, the first page of which requested their consent to allow the survey results to be used for research purposes and to allow researchers to analyze their coursework, including exams. Consent rates were 56\% for Instructor A, 93\% of Instructor B, and 96\% for Instructor C. We were missing complete final exam data for 3 students, which brought final participant numbers to $\mathrm{N}_{\mathrm{A}}=77, \mathrm{~N}_{\mathrm{B}}=133$, and $\mathrm{N}_{\mathrm{C}}$ $=137$.

Research Questions. Between the three instructors, we were centrally concerned with two comparisons. The first was between Instructors A and B, who differed in (i) teaching experience, with A highly experienced and B a novice, and (ii) instructional emphasis, with A focused on traditional problem solving and B focused on mathematical sense-making. We hypothesized that these differences might lead to a trade-off in which Instructor A outperforms Instructor B with respect to teaching students traditional problem-solving, while B outperforms A with respect to teaching students mathematical sense-making. This comparison is the most relevant for instructors considering PER-based strategies, because in evaluating the $1^{\text {st }}$-time use of PER-based instruction, instructors and departments typically compare the PER-based class to well-established traditional classes. By comparing students' responses to the common final exam questions in the two instructors' sections, we aimed to answer the following:

\section{Research questions comparing Instructors $A$ and $B$ :}

- Can a novice instructor's explicit emphasis on mathematical sense-making successfully support mathematical sense-making in a large lecture physics course?

- As compared to an experienced "traditional" instructor, can a novice instructor's focus on mathematical sense-making lead to diminished success at inculcating "traditional" problem solving methods?

By contrast Instructors $\mathrm{A}$ and $\mathrm{C}$ were both experienced, enabling us to compare the effects of polished versions of the two instructional emphases:

\section{Research questions comparing Instructors $A$ and $C$ :}

- As compared to instruction focused on traditional problem solving, can instruction focused on mathematical sense-making increase students' success at using such reasoning?

- Is it possible for instruction focused on mathematical sense-making to inculcate "traditional" problem-solving skills comparable to instruction focused on traditional problem-solving?

We now describe how we addressed these research questions.

Final exam construction and coding: Overview. The three instructors wrote a single final exam taken by students from all three lecture sections. Our analysis in this paper focuses on the freeresponse questions, which were the majority of the exam. The free-response portion of the exam consisted of three problems divided into a total of 13 parts.

Before coding students' answers, we divided questions into two types. We labeled three parts (one from each problem) as sense-making opportunity questions because, like the problem about 
the thrown vs. dropped ball, they could be answered quickly without calculations if the student employed mathematical sense-making. These sense-making opportunities could also be solved in a calculation-intensive way, and our coding scheme - discussed below-distinguished whether students attained the correct answer through mathematical sense-making or a more calculational approach.

We labeled the other problems as traditional because they were most naturally and efficiently solved using the expert traditional problem-solving approach discussed above. Of course, students could incorporate a greater or lesser degree of mathematical sense-making into their solutions to these traditional problems. But we did not expect this mathematical sense-making to be clearly visible in their written exam solutions. The sense-making opportunity questions, by contrast, are the ones for which we thought students' responses could provide clear evidence of mathematical sense-making.

We coded students' responses to both of these question types. The traditional problems were coded for correct approach; a response was coded as "correct" if the student used the correct physical principle with correct values substituted into the relevant equation, even if the student made a minor algebraic or arithmetic error leading to an incorrect final answer. We did not double count errors consequent from an incorrect answer on a previous part of the problem. We also coded which answers were fully correct, and found that using our coding scheme vs. "full correctness" did not affect which instructors' students outperformed each other, as reported below.

Responses to the sense-making opportunity questions were coded for whether students' correct answers were at least partly supported through mathematical sense-making. Specifically, in these three sense-making opportunities, the mathematical sense-making approach involves the correct use of conceptual or heuristic reasoning in a solution that could be used to avoid explicit calculations. This means the mathematical sense-making code excludes both (i) incorrect answers and (ii) correct answers supported only by explicitly worked out computations. Since our data come from a final exam, we expected that many students would do explicit calculations even if they thought of a quick, heuristic answer, in order to get "full credit" or to be sure of their answers. Therefore, we coded answers as reflecting mathematical sense-making if any part of a student's solution included mathematical sense-making, whether or not the student also did a calculation. The details of the sense-making coding on each problem are described in the next sub-section.

Our preliminary coding scheme was generated by three of the authors by looking at a small subset of the student responses $(\mathrm{N}=25)$. Two authors then coded $45 \%$ of the students' responses, distributed proportionally across the 3 instructors. The two coders agreed on $93 \%$ of the traditional codes (Cohen's $\kappa=.86$ ) and $96 \%$ of the sense-making codes (Cohen's $\kappa=.89$ ). After resolving disagreements and refining the coding scheme, one coder then coded the remaining responses. After this coding was finished, discussion between the authors led to the addition of a solution category to the sense-making code on the exploding blocks problem. One coder recoded the exams according to this modification, which led to 12 changes in coding (3.5\% of exploding blocks sense-making codes). The second coder "trained" on 2 of these 12 changes, then recoded the other 10 independently, agreeing with the first coder on 9 of the 10 cases. 
Coding the sense-making opportunities. We now walk through the exam problem-by-problem, identifying which parts we labeled as a sense-making opportunity and illustrating how we coded for a mathematical sense-making vs. a calculational response. Appendix A provides our detailed coding scheme.

\section{EXAM QUESTION 1: EXPLODING BLOCKS}

Two identical masses, each of mass $\mathrm{m}=50 \mathrm{~g}$, are fastened to each other with a bit of plastic explosive. We're going to launch it into the air and detonate the explosive at the highest point. (Ignore air resistance throughout this problem.)

(a) Suppose we launch the pair of masses at an angle $\theta=60$ degrees above the horizontal, from a spring gun. The spring has a spring constant of $k=1000 \mathrm{~N} / \mathrm{m}$, and we compress it $x=10 \mathrm{~cm}$. $\left(\sin 60^{\circ}=0.87\right.$; $\cos 60^{\circ}=0.5$ ). Find the maximum height of the pair of masses, taking its initial height to be 0 .

(b) At exactly that instant, when it's at the highest point, we detonate the explosive. And it so happens that the instant after the explosion, one mass (A) is not moving at all. Find the velocity of the other mass (B).

(c) Find the distance between the masses A and B when they hit the ground.

(d) During the explosion, mass B speeds up while mass A comes momentarily to rest. Does the overall mechanical energy of the two-mass system increase, decrease, or stay the same during that explosion? Explain.

(e) Sketch a graph of the vertical and horizontal components of the velocity for mass B from the time of launch to the time it hits the ground. Explain your reasoning. You don't need to make precise calculations, just show the shape of the graph in your sketch.

\section{FIGURE 1. Exam question 1}

Figure 1 shows the first free-response exam question. We categorized part (d) as a mathematical sense-making opportunity, because a student can avoid calculations by connecting the commonsense insight or conceptual realization that an explosion speeds things up, or that the nonmechanical energy "lost" in the explosion must go into the blocks, to the equation $\Delta E_{\text {mechanical }}=$ $\Delta K+\Delta U$, where in this case the potential energy $U$ does not change during the explosion. Thus, the student response shown in figure 2 was coded as mathematical sense-making; the student leverages a conceptual argument about energy conservation to answer the question without appealing to an explicit calculation.

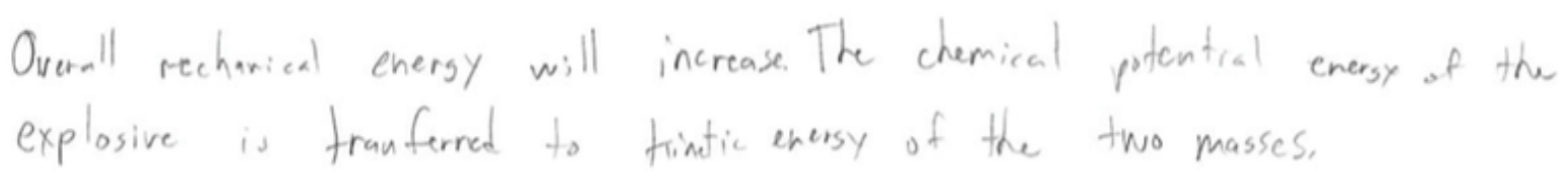

Figure 2. A response to Exploding Blocks part (d) coded as mathematical sense-making 
For this problem, a second subcategory of mathematical sense-making consisted of some variant of the following reasoning: By momentum conservation, the mass of the moving part of the system gets cut in half, while its speed doubles. In the expression for kinetic energy, $1 / 2 m v^{2}$, the velocity is squared, which means the increase in $v$ more than compensates for the decrease in $m$, and hence the kinetic energy increases. We coded this reasoning as sense-making because the student appeals to functional relations in the expression for kinetic energy to take a "shortcut" past explicit calculation and comparison of the pre- and post-explosion kinetic energies.

By contrast, figure 3 is a correct answer we did not code as mathematical sense-making.

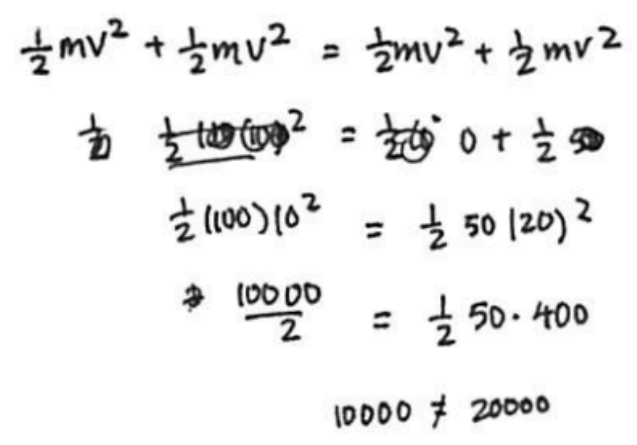
overall mechanical energy increases, as the initial kinetic energy of the systan was only 10,$000 ;$ betore the explosion, while atterwerds it is $20,000 \mathrm{j}$.

Figure 3. A response to Exploding Blocks part (d) not coded as mathematical sense-making.

Although this solution uses the wrong units for mass and adopts an incorrect value of $v$ computed incorrectly in a previous part of the problem, we would consider this student's response to be correct, because it represents a correct approach. However, this answer is not coded as mathematical sense-making because the student performs an explicit calculation without appeal to a conceptual reason for why the energy must increase.

Note that even logically incomplete responses may be coded as mathematical sense-making if there is the presence of physical reasoning that could lead to a correct result . For instance, consider this response:

The overall mechanical energy increases because the explosion adds an outside force to the system adding energy to the system.

This answer is incomplete: an external force could also slow a system down, taking away energy. A fully correct version of this answer would need to include an argument for why the work done by the forces on the system is positive. Still, though not proven, the answer represents correct physical reasoning about the process, how the explosion pushes on the blocks, and a connection to the mechanical energy to attain the correct result. So, we coded it as mathematical sensemaking. 
In developing the coding scheme for this item, we iteratively cycled through the responses, creating fine-grained categories of responses, and then decided which of those categories counted as mathematical sense-making. The above examples illustrate the kind of reasoning we went through in making these decisions.

The second free-response question is shown in Figure 4.

\section{EXAM QUESTION 2: BALLISTIC PENDULUM}

A uniform rod of length $L(=1.00$ meter $)$ and mass $M(=1.80 \mathrm{~kg})$ is hanging vertically from a frictionless pivot at its top end. A bullet of mass $m(=400 \mathrm{gm})$ strikes the rod at the center of the rod and gets embedded in it. (See figure.) Right at the instant before the bullet hits the rod, the velocity of the bullet was entirely horizontal (and perpendicular to the rod) and the magnitude of the bullet's velocity was $v(=100 \mathrm{~m} / \mathrm{s})$. You can imagine that the rod with the embedded bullet would rotate about the pivot. The moment of inertia of the rod about the pivot is $\mathrm{ML}^{2} / 3$.

(a) Is this an elastic or an inelastic collision? How do you know?

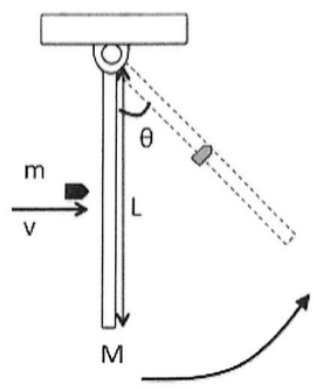

(b) Solving for the angular speed of the rod with the embedded bullet immediately after the collision, a student comes up with this answer: $\omega=\frac{3 M V}{2 m L}$. Is that a plausible answer? Explain your reasoning to her without solving for that angular speed yourself.

(c) Now solve for the angular speed of the rod with the embedded bullet immediately after the collision.

(d) What is the ratio of the kinetic energy of the rod and bullet system after the collision to that of the bullet before the collision?

(e) Will the rod hit the ceiling? Explain.

Figure 4. Exam question 2.

We categorized part (b) as a mathematical sense-making opportunity, because the question invites students to examine proportionalities and inverse proportionalities in the equation to "read" the physical meaning of the equation and thereby examine its plausibility. Such reasoning is mathematical sense-making because it involves associating physical meaning with the functional form of the equation, instead of viewing the equation as merely a black box for calculating answers. For instance, one student wrote

Based on this equation, the heavier the rod is, the faster the final speed will be. That doesn't make sense physically.

We infer that the student notices that, according to the equation, the angular speed $\omega$ is proportional to the rod's mass, $M$, which would mean a heavier rod corresponds to less angular speed after the bullet hits it. The student concludes, correctly, that this "doesn't make sense physically." 
Some responses could have reflected either traditional problem solving or mathematical sensemaking. For instance, one student wrote,

This equation doesn't work, because the bullet and the rod stick together. When you calculate the momentum, it should have the masses added together, $(m+M)$.

The student might have reached this conclusion by realizing on conceptual grounds that the angular speed after the collision should depend on the combined mass of the bullet+rod system and noticing that the equation does not encoded this insight - an example of mathematical sensemaking. By contrast, the student might have used traditional problem solving to calculate the answer in part (c), noticed that the answer contains " $m+M$," and decided that the part (b) equation doesn't work because it lacks that expression. This excellent but purely formal/symbolic reasoning is not mathematical sense-making because it does not involve integration of conceptual and formal reasoning; it's purely formal, relying on a formal calculation or derivation instead of using a conceptual insight to avoid a calculation. Because we cannot tell from the second student's answer whether it stemmed from mathematical sensemaking or from purely formal reasoning, we did not code it as mathematical sense-making.

Figure 5 shows the third free-response exam problem.

\section{EXAM QUESTION 3: RAMPS}

A block of mass $M$ sits on a ramp of angle $\theta$.

(a) First, suppose the block is frictionless and is held in place by a light string extended parallel to the surface of the ramp, as shown here. Write an expression for magnitude of the tension in the string.

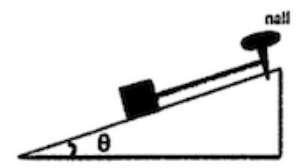

(b) Instead of a peg, we have the cord connect over a pulley to another block. The second block is just the right mass so that the first block remains at rest. Write an expression for the magnitude of the tension in the string.

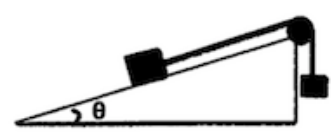

(c) Now, suppose there's no string, but the block stays in place because of friction (with coefficient of static friction $\mu$ ) between the block and the ramp. Write an expression for magnitude of the friction force by the ramp on the block.

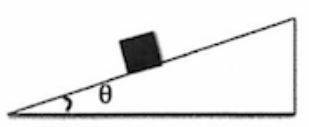

Figure 5. Exam question 3.

Here, mathematical sense-making leads to the realization that these three situations are physically equivalent in an important sense: a force directed up the ramp balances the component of gravity pulling the block down the ramp. Therefore, the force directed up the ramp must be the same in each case, $m g \sin \theta$. In other words, having solved part (a), a student engaged in mathematical sense-making can just write down the answers to (b) and (c) without further calculations. We coded a student's response as mathematical sense-making if the student showed evidence of taking this shortcut on at least one of parts (b) and (c). For instance, one student answered (b) and (c) as follows: 


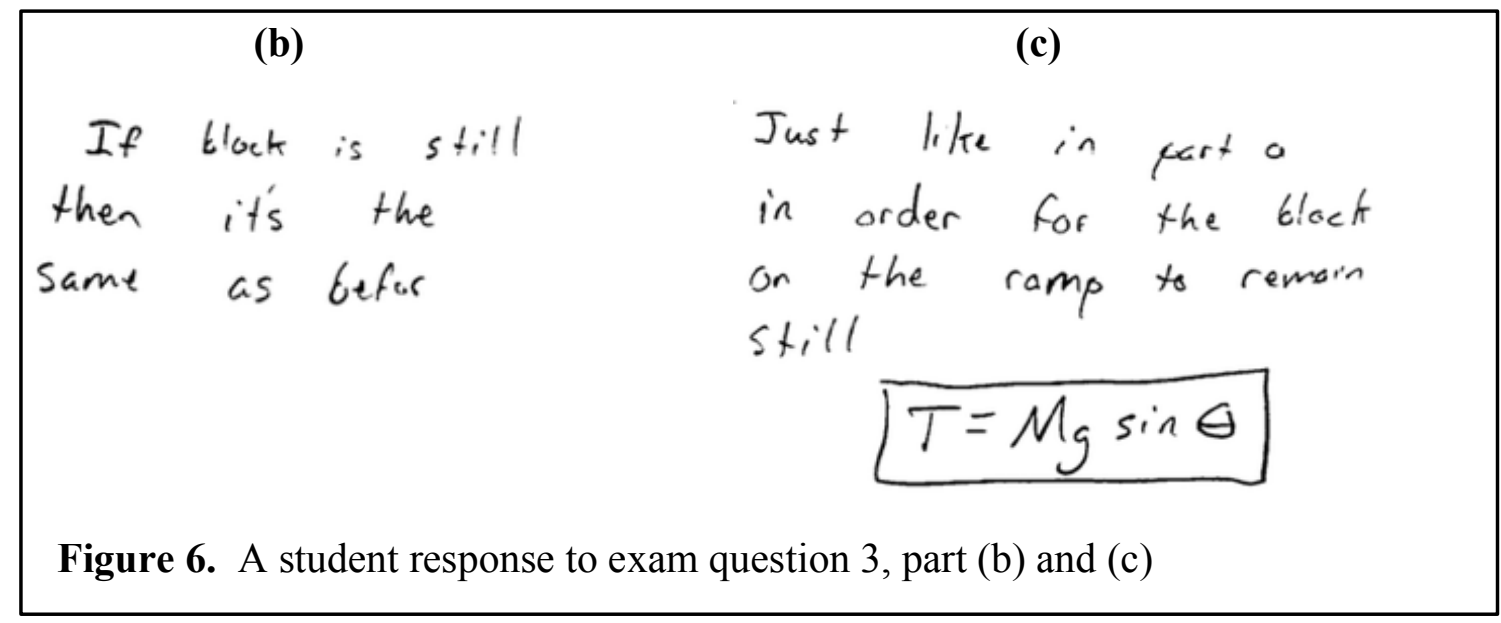

In other cases, it was clear that the student was treating each of the three parts separately without realizing they must have the same answer. In several cases, however, it was hard to tell whether or not the student had made that realization. For instance, consider Figure 7, an idealization of some student responses:

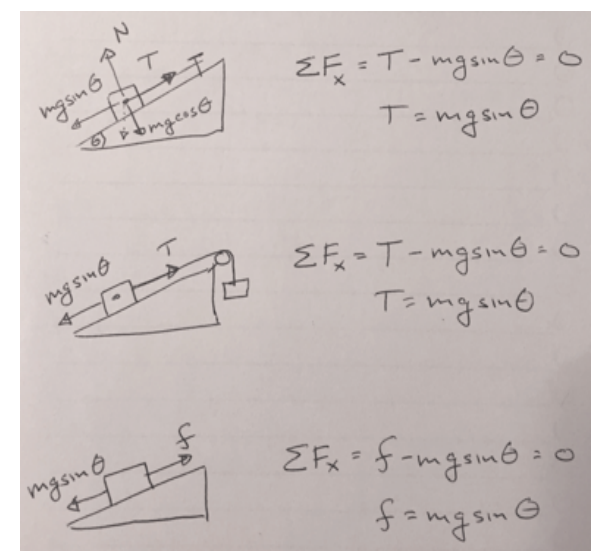

Figure 7. A response to exam question 3 that would not be coded as mathematical sense-making.

Here, the hypothetical student might understand that all three answers must be the same but nonetheless recalculates the answer each time to ensure full credit. Or, the hypothetical student could be working through each problem without realizing that they are all the same problem and the answers must come out the same. When a student (as in Fig. 7) did not explicitly indicate his or her realization of the relevant physical equivalence of the three scenarios, we did not code the response as mathematical sense-making.

\section{Results}

Recall that A is the experienced, well-regarded "traditional" instructor, B is the novice instructor who attempted to emphasize mathematical sense-making, and $\mathrm{C}$ is the experienced instructor and physics education researcher, who also emphasized mathematical sense-making. 
Mathematical sense-making: We find each student's total mathematical sense-making score by summing his or her scores on each separate sense-making opportunity. So, students' scores range for 0 to 3 . Figure 8 shows the distributions of total sense-making score by instructor.

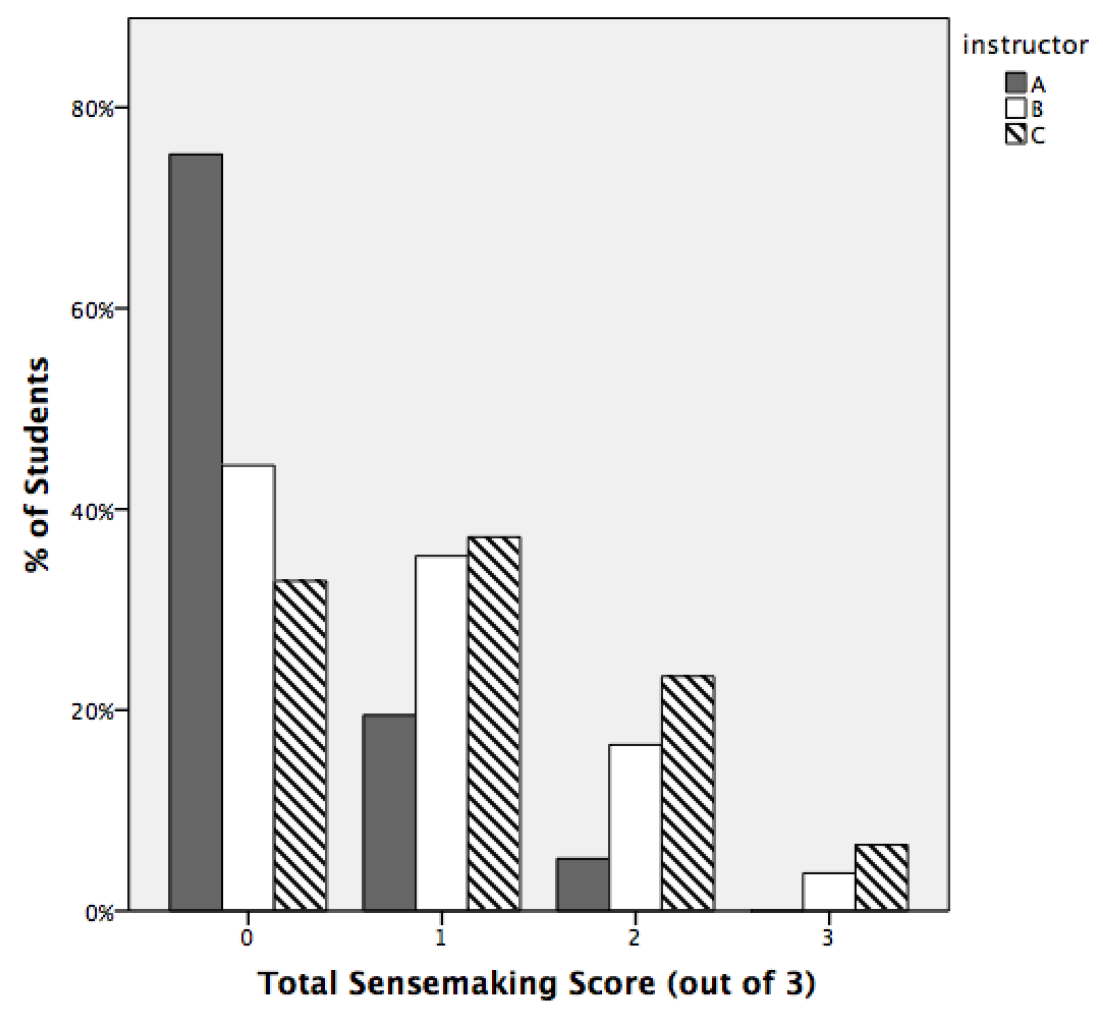

Figure 8. The distribution of total sense-making scores (ranging from 0 to 3 ) for each instructor.

Looking at what percentage of students gave zero sense-making answers shows the general trend: $75 \%$ of Instructor A's students, having experienced instruction emphasizing traditional problem solving, gave no sense-making responses on the final exam. By contrast, only $44 \%$ of Instructor B's students and 33\% of Instructor C's students, who experienced instruction emphasizing mathematical sense-making, gave no sense-making responses.

Because the distributions are highly skewed, we compared the rank-order of student total sensemaking scores for each instructor. A one-way Kruskal-Wallis test reveals a significant difference in the mean ranks for the three instructors, $\mathrm{H}(2)=38.5, \mathrm{p}<.001$ ), with a mean rank of 120 for Instructor A, 177 for Instructor B, and 202 for Instructor C.

Pairwise comparisons of rank-order were done with the Mann-Whitney U test at the Bonferronicorrected $\alpha=.05 / 3$. In our planned comparisons of total sense-making score, Instructor B's class outperforms Instructor A's, $\mathrm{Z}=4.49, \mathrm{p}<.001$, and Instructor C's class also outperforms Instructor $A$ 's, $Z=6.13, p<.001$. These results show that both a novice and an experienced instructional focus on mathematical sense-making do better than traditional instruction at fostering students' sense-making. 
The rank-biserial correlation $r$ is one measure of the effect size of the difference in ranks. It represents how often (above chance) a randomly selected value from one distribution will be greater than a randomly selected value from the other. Therefore, two identical distributions will have an $r=0$ and two non-overlapping distributions will have an $r=1$. For the comparisons above, $r_{\mathrm{AB}}=.33$ and $r_{\mathrm{AC}}=.47$.

Comparison between Instructor B's and Instructor C's classes shows no significant difference in rank ordering of total sense-making score, $Z=2.22, p=.03$. Said another way, there is no statistically significant difference between a novice and experienced emphasis on sense-making, although the trend favors experience.

None of the professors' students showed as much facility with mathematical sense-making as we would have liked: $47 \%$ of all students scored 0 and $33 \%$ scored a 1 . It is important to note, however, that these students knew they would receive full credit for correct answers based on calculations. We also note that Physics 1 is the first of a three-course sequence. We have noticed anecdotally that students who took multiple physics courses emphasizing mathematical sensemaking displayed further progress toward facility and comfort with such sense-making, beyond what was achieved in this first semester.

Traditional problems: For the 9 traditional problems, we summed each student's score ( 0 or 1$)$ on each part to arrive at a Traditional Total score between 0 and 9 . See appendix B for details on how we coded. Figure 9 shows the results.

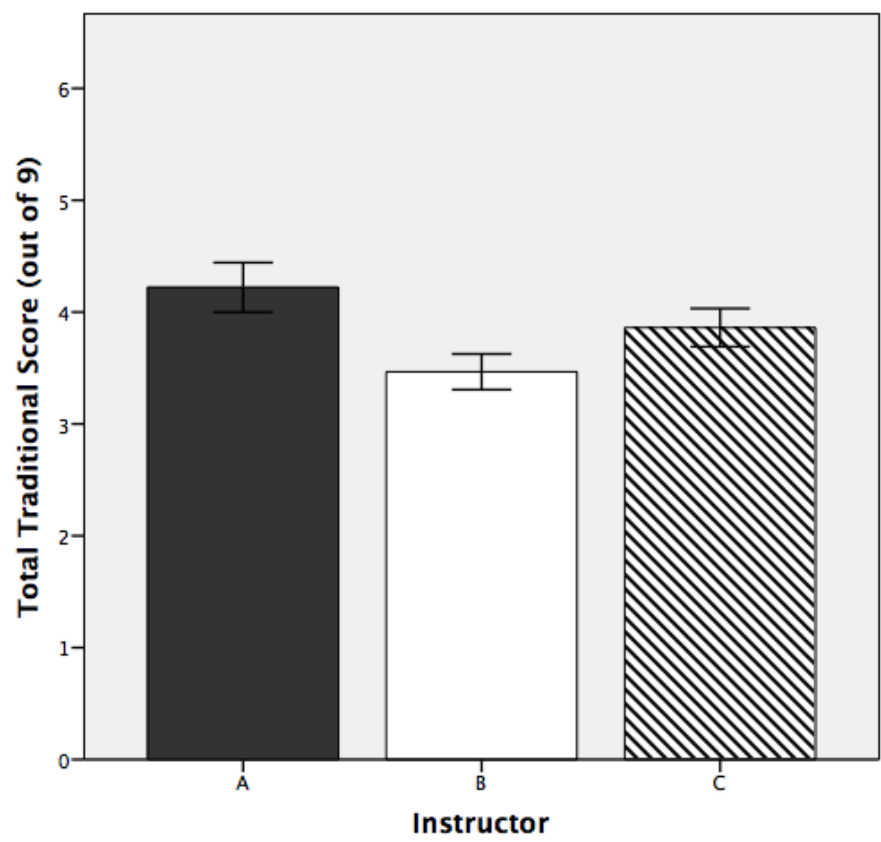

Figure 9. The total traditional problem-solving scores for each instructor. Error bars represent 1 standard error. 
Compared to the sense-making score distributions, the traditional score distributions were more normal, and we therefore compare the mean traditional scores for the three instructors. Considering the three instructors together, there is a difference in the means of the total traditional score, $\left(\mathrm{m}_{\mathrm{A}}=4.22, \mathrm{sd}_{\mathrm{A}}=1.94, \mathrm{~m}_{\mathrm{B}}=3.47, \mathrm{sd}_{\mathrm{B}}=1.84, \mathrm{~m}_{\mathrm{C}}=3.86, \mathrm{sd}_{\mathrm{C}}=2.00\right), \mathrm{F}(2,344)$ $=3.89, \mathrm{p}=.02$. Post-hoc testing with the Games-Howell test shows that the only pairwise difference is Instructor A's students outperforming B's, $\mathrm{p}=.02, \mathrm{~d}=.40$. An experienced focus on traditional problem solving does foster traditional problem-solving skill better than a novice focus on mathematical sense-making does.

Although we do not know if novice traditional instruction would have matched Instructor A, our result does show that novice instruction successful in fostering mathematical sense-making does not guarantee the fostering of traditional problem solving to the level of polished traditional instruction. However, there is no difference between Instructor A's and C's students on this measure of traditional problem solving, $p=.41$. This suggests that, though not guaranteed, an instructor experienced with PER-based pedagogy can emphasize mathematical sense-making without a loss in traditional problem-solving success.

Comparison between Instructor B's and Instructor C's classes shows no significant difference in total traditional score, $\mathrm{p}=.21$; there is no difference between a novice or expert focus on mathematical sense-making in terms of traditional problem-solving outcomes.

\section{Discussion and Conclusion}

Our results do not inform discussions about whether traditional problem solving or mathematical sense-making is a worthier instructional target for introductory physics classes aimed at engineering majors. By contrast, our results do provide an existence proof that, at least for novice instructors, these goals can be in tension. By focusing on fostering students' mathematical sense-making, Instructor B enabled his students to outperform Instructor A's students at mathematical sense-making. But this instructional success came at a cost; Instructor B's students underperformed on the traditional problems. We now discuss the implications of these results for instructors and researchers.

Implications for researchers. Given our existence proof that such instructional trade-offs can exist between mathematical sense-making and traditional problem solving, researchers could investigate under what conditions that trade-off is appreciable. Is it only for novice instructors? For all but the most experienced and dedicated instructors?

This research also motivates a closer look at potential trade-offs between other pairs of instructional goals. Previous work hints at but doesn't systematically explore such trade-offs and disconnects. For instance, Kanim ${ }^{18,19}$ found that even students who displayed good conceptual understanding of electric circuits as indicated by conceptual exams did not apply their qualitative understanding when solving standard quantitative circuit problems. In other words, they didn't engage in mathematical sense-making using the conceptual knowledge they possessed. This suggests a possible trade-off between maximizing qualitative conceptual understanding and maximizing mathematical sense-making. 
Researchers could also conduct studies in which students are taught physics in different wayse.g., with an emphasis on mathematical sense-making vs. traditional problem solving - and then followed into subsequent engineering courses. Are there differences in how the students approach learning or problem-solving, and do they perform differently? Such research, rather than "philosophical" debates about what engineering students need to get out of their physics courses, should drive reforms in introductory physics courses for engineers.

Finally, both researchers and journals need to resist publication bias that can lead to instructional trade-offs remaining underground.

Implications for instructors. In our roles as instructors, we have attended many course/instructional committee meetings at which different instructors suggest emphasizing two different instructional targets. The result is almost always "let's emphasize both!" However, if the results of this study are not idiosyncratic, then given time constraints, trade-offs must be made.

This mindset has the potential to change how we evaluate the successes of first-time attempts at course reforms. If we introduce a new instructional emphasis without expecting set-backs in other instructional goals, any successes of the new teaching method might be overshadowed by set-backs elsewhere. By contrast, if we expect successes with respect to the new instructional emphasis to come with a trade-off, we might be more inclined to allow the new style of instruction to mature. Studies such as this one illustrate that trade-offs associated with implementing new types of instruction, as illustrated by Instructor B, can fade as the instruction becomes more polished over time, as illustrated by Instructor C. First-time instructors (and their colleagues) should not expect everything all-at-once.

Engineering design embraces the idea of such trade-offs. We encourage instructors to frame course design as a kind of engineering (or at least as analogous to engineering), so that such trade-offs - and the multiple strategies engineers have for dealing with them-come to the foreground in instruction.

\section{Acknowledgments}

We thank David Hammer for helpful comments. This work was supported by National Science Foundation Award 0835880.

\section{References}

1. McDermott, L.C., \& Redish, E.F. (1999). Resource Letter PER-1: Physics Education Research. American Journal of Physics, 67, 755-767.

2. Meltzer, D.E., \& Thornton, R.K. (2012). Resource letter ALIP-1: active-learning instruction in physics. American journal of physics, $80,478$.

3. Prince, M. (2004). Does active learning work? A review of the research. Journal of engineering education, 93(3), 223-231. 
4. Gainsburg, J. (2006). The mathematical modeling of structural engineers. Mathematical Thinking and Learning, 8(1), 3-36.

5. Hall, R., \& Stevens, R. (1994). Making space: A comparison of mathematical work in school and professional design practices. The Sociological Review, 42(S1), 118-145.

6. Hake, R.R. (1998). Interactive-engagement vs traditional methods: A six-thousand-student survey of mechanics test data for introductory physics courses. American Journal of Physics, 66(1), 64-74.

7. Chasteen, S.V., Pollock, S.J., Pepper, R.E., \& Perkins, K.K. (2012). Transforming the junior level: Outcomes from instruction and research in E\&M. Physical Review Special Topics-Physics Education Research, 8(2), 020107.

8. Lasry, N., Mazur, E., \& Watkins, J. (2008). Peer instruction: From Harvard to the two-year college. American Journal of Physics, 76(11), 1066-1069.

9. Hoellwarth, C., Moelter, M.J., \& Knight, R.D. (2005). A direct comparison of conceptual learning and problem solving ability in traditional and studio style classrooms. American Journal of Physics, 73(5), 459462.

10. Eylon, B.-S., \& Reif, F. (1984). Effects of knowledge organization on task performance. Cognition and Instruction, 1, 5-44.

11. Reif, F. (1983). Understanding and teaching problem solving in physics. Proceedings of the first international workshop: Research on Physics Education. La Londe les Maures.

12. Reif, F., \& Larkin, J.H. (1991). Cognition in scientific and everyday domains: comparison and learning implications. Journal of Research in Science Teaching, 28(9), 733-760.

13. Docktor, J., \& Heller, K. (2009). Assessment of student problem solving processes. Paper presented at the 2009 Physics Education Research Conference.

14. Heller, P., \& Hollabaugh, M. (1992). Teaching problem solving through cooperative grouping. Part 2: Designing problems and structuring groups. American Journal of Physics, 60(7), 637-644.

15. Heller, J.I., \& Reif, F. (1984). Prescribing effective human problem-solving processes: Problem description in physics. Cognition and Instruction, 1, 177-216.

16. Larkin, J., McDermott, J., Simon, D.P., \& Simon, H.A. (1980). Expert and novice performance in solving physics problems. Science, 208(4450), 1335-1342.

17. Kuo, E., Hull, M., Gupta, A., \& Elby, A. (2013). How students blend conceptual and formal mathematical reasoning in solving physics problems. Science Education, 97(1), 32-57.

18. Kanim, S. (2011). Physics Education Research: Adapting to new topics and student populations. Bulletin of the American Physical Society, 56.

19. Kanim, S. (1999), An investigation of student difficulties in qualitative and quantitative problem solving: Examples from electric circuits and electrostatics. Doctoral dissertation in Physics, University of Washington: Seattle. 


\section{Appendix A: Coding scheme for the mathematical sense-making opportunities}

The following is the actual document that the two coders used, as it evolved over the iterative refinement of the scheme.

\section{Overall META-NOTES on Coding}

- Coding must be done in order. That is, you can't look at part $\mathrm{c}$ to inform your interpretation of part a. One reason is that we already look at part a to inform part c, when not trying to "double count" errors that happened in earlier parts. If we allow also looking forward, then students may get a perfect score, even though there is an error.

- Pure symbolic answers are acceptable, so long as it seems like students conceptually understand what's going on. The numbers aren't required, although they can provide evidence that the student is making some conceptual error. A sloppy plug-in of a value can still be coded as correct, if it seems clear that the student conceptually understands what to do.

PART II:

Coding for "sense-making"

A more detailed description of what this means for each part of each problem that is coded here:

- $\quad$ On the exploding masses problem:

- For part (d), student answers correctly that the total energy of the two-mass system increases and gives a conceptual reason why this makes sense (i.e. students don't answer only from an explicit, numerical calculation). These "conceptual" reasons include:

- The "explosion" increases energy

- external work is done

- an external force exists

- chemical energy was turned into mechanical energy

- OVERALL POINT: We are looking for the conceptual idea that the explosion adds energy and care less about whether the technical details are correct. If a student says that "the explosion adds kinetic energy to the system" that should be coded as "sense-making," because students are thinking about the conceptual effect of the explosion and not just quoting the rule that energy is always conserved.

- $\quad 1 / 2$ point: Students who say "Energy is conserved. The energy lost by one block is gained by the other." This is sense-making in that it makes an argument using conservation of energy to argue for why it makes sense that one block loses energy.

- Mathematical Sense making with the equation:

- Responses are coded as sensemaking if they give the following explanation: Although the mass is cut in half, the speed doubles. Since $K E \sim v^{2}$, the velocity has a bigger impact on the KE, so the final energy increases."

- Just saying that velocity depends on $\mathrm{v}^{2}$ is not enough. Sensemaking answers must acknowledge that mass also decreases (i.e. acknowledging competing factors).

- On the ballistic pendulum problem: 
- For part (b), students interpret the meaning of the mathematics to see if the expression makes sense:

- Code 1a: Comparing initial and final linear or initial to final angular velocities to check against what we expect to happen: Students must compare linear to linear or compare angular to angular. No comparisons of linear to angular.

- Code 3a: thinking correctly about dependencies - whether variables are directly or inversely proportional to angular speed and whether those dependencies make conceptual sense. Students who incorrectly thought about dependencies were coded as "not sense-making".

- Many students try to solve this problem by coming up with their own solution for or imagining what would be involved in solving for omega. This is not sufficient evidence to count as sense-making about the expression.

- Metanote about sense-making coding here: We want to capture whether we think students are making sense of relationships vs. whether they are doing unit analysis or trying to derive the equation from other formulas. If we think that they are thinking of these relationships, even though they are not well articulated, then we should code it as "sensemaking" since that's the mode we suspect students were in (so long as the reasoning is also correct). This also strengthens our argument, since we might expect Prof. A's students to not be as eloquent at expressing correct conceptual reasoning since they don't have as much practice. With this coding scheme, we allow such students who "know it but can't articulate it well" to be counted.

- On the Ramps problem:

- For parts (a) and (b), students explicitly note that these two problems are conceptually identical and because of that should have the same answer (ex. If a student derives the solution in the same way in $b$ as in a, this is not sufficient evidence for a sense making code. A student must explicitly note that the solution to part $b$ is the same as in $a$.)

- For part (c), students explicitly note that this problems is conceptually identical to part (a) or (b) and because of that should have the same answer (ex. If a student derives the solution in the same way in $\mathrm{c}$ as in a, this is not sufficient evidence for a sense making code. A student must explicitly note that the solution to part $\mathrm{c}$ is the same as in a.)

\section{Appendix B: Coding scheme for the traditional problems parts}

\section{PART I: \\ Coding for "correctness:" Do students implement the correct concepts and equations?}

This is a strict coding analogous to what would count as correctness for a typical physics course:

- $\quad$ On the exploding masses problem:

- For parts (a) - (c), did the student implement the correct physics concept? This means they started with the right generic physics concept and made clear that they understood the meaning of the symbols (so they associated the correct variable labels or numerical values to the different quantities in the equation). Errors in the final answer from math errors are ok, but you need to choose accurate and appropriate physics principles and plug in the correct numbers/quantities.

- (a) Do students use correct physics to find the maximum height of the peak?

- Although technically part of the change in height, students are not required to take into account the change in height from the decompression of the spring to be coded as correct. 
- Trigonometric errors are coded as correct if it is clear that the student is conceptually solving for the correct vector component (ex. If students correctly seek to solve for the vertical component of velocity but incorrectly tries to solve for the vertical component with cosine, then this is coded as correct.)

- (b) Do students use conservation of momentum or some other correct physics principle?

- (c) Do students use correct kinematic concepts or some other correct physics principle?

- For part (e), students made the correct graphs.

- On the ballistic pendulum problem:

- For part (a), student answers "inelastic collision" or "energy is lost/ not conserved" with a correct explanation of why that makes sense (ex. bullet gets stuck, doesn't bounce off, sound and heat from colliding and sticking, bullet does work to get lodged in there.)

- For part (c), student uses conservation of angular momentum (not conservation of energy or linear momentum). Calculation errors are ok, but it must be clear that correct conceptual ideas and symbolic expressions must be there (same criteria as above). Importantly, I_final must be the moment of inertia of the rod + the moment of inertia of the bullet

- Students must use correct I_final exactly to be coded as correct (correct formula with evidence that you plugged in the conceptually correct values)

- For part (d), to be coded as "correct", a solution must meet the following two criteria:

- 1) Express the initial kinetic energy as $1 / 2 \mathrm{~m}_{\text {bullet }} \mathrm{v}_{\text {bullet }}$, even if the numerical value is not calculated correctly

- 2) Express the final kinetic energy as $1 / 2 \mathrm{Iw}^{2}$, with a clear indication of $\mathrm{I}$ and $\mathrm{w}$ being the value found in part $\mathrm{c}$ (even if part $\mathrm{c}$ is incorrect or blank). Again, the numerical value need not be calculated correctly.

- Incorrect expressions for moment of inertia are OK, only if it is consistent with previous parts of the problem.

- It is also OK if the student does not find numerical values for the kinetic energies. Finally, although the problem asks for a ratio of the kinetic energies, this was not necessary to be coded as "correct". It is sufficient to correctly find expressions for the initial and final KE.

- For part (e), student calculates potential energy as mgh, where $\mathrm{m}$ is the total mass $(1.8+$ $.4 \mathrm{~kg}$ ) and $\mathrm{h}$ is the distance from the center of mass of the rod+bullet system to the ceiling $(0.5 \mathrm{~m})$. The values for KE must be consistent with part (d), even if incorrect.

- On the Ramps problem:

- For part (a), did the student set up their answer to this question correctly (Is the answer correct? If not, is the conceptual set up correct, meaning that trigonometric errors in calculating components are ok if it's clear that students are trying to find the correct component of the force for N2)

- For part (b), did the student set up their answer to this question correctly? (mg*sin(theta) is the correct answer.

- Again, a trig error is ok, if there is evidence from diagrams or symbols that the student is attempting to calculate the component of weight down the ramp.

- $\mathrm{m} \_$hanging $* \mathrm{~g}$ is not correct, because it is not in terms of the given information in the problem. Students may say that the Tension in the rope is equal to both of these. This is ok, since both are technically correct statements, but a correct code means that $\mathrm{mg}$ *sin(theta) has to be there.) 
- For part (c), did the student set up their answer to this question correctly? (mg sin(theta) is the correct answer.

- Like kinetic coefficients of friction, static coefficients of friction were taught to students as being a constant, such that the static friction force is less than or equal to $(\mathrm{mu}) * \mathrm{~N}$. Hence, students who answered $(\mathrm{mu}) * \mathrm{~N}$ for this question were liable to have points deducted by the grader of the final exam. However, for this coding, if a student BOTH said that friction is equal to (mu)*N AND that one of those two expressions is equal to mgsin(theta), the student was counted as being correct. If the student only lists (mu) $\mathrm{N}$ as the answer, then it is coded as incorrect

- (Special Case: If a student writes that F_friction= mg sin (theta), but then proceeds to solve for mu, then it's coded as incorrect, because the student didn't know what the question was. If they do this, but also box the F_friction and/or mg $\sin ($ theta), then that is coded as correct.) 